\title{
Japanese Kampo medicine ninjin'yoeito synergistically enhances tumor vaccine effects mediated by $\mathrm{CD8}^{+} \mathrm{T}$ cells
}

\author{
SHUN TAKAKU, MASUMI SHIMIZU and HIDEMI TAKAHASHI \\ Department of Microbiology and Immunology, Nippon Medical School, Tokyo 113-8602, Japan
}

Received April 25, 2016; Accepted February 28, 2017

DOI: $10.3892 / \mathrm{ol} .2017 .5937$

\begin{abstract}
Although Japanese traditional herbal medicine (Kampo) has been widely applied to the treatment of various diseases, including cancer, their mechanisms of action have not yet been elucidated in detail, particularly regarding their role in tumor immunology. The present study investigated the antitumor effects of the Japanese Kampo medicine, ninjin'yoeito (NYT; Ren-Shen-Yang-Rong-Tang in Chinese), which was orally administered with or without an irradiated tumor cell vaccine to a subcutaneous CT26 colon carcinoma tumor model. The irradiated tumor cell vaccine in a prophylactic setting significantly delayed tumor growth in mice fed a control diet, whereas a diet containing NYT alone did not exert any antitumor effects in vivo. However, the inhibition of tumor growth was significantly greater in vaccinated mice fed the NYT diet compared with in vaccinated mice given the control diet. These results suggest that NYT synergistically enhances the effects of the antitumor vaccine. The depletion of cluster of differentiation (CD) $8^{+} \mathrm{T}$ cells abrogated these effects, indicating that antitumor activity required $\mathrm{CD} 8^{+} \mathrm{T}$ cells. Furthermore, reductions in $\mathrm{CD} 4^{+} \mathrm{CD} 25^{+}$and forkhead box protein $3^{+} \mathrm{T}$ regulatory cell numbers were more apparent between vaccinated mice fed the NYT diet and non-vaccinated mice fed the control diet than between vaccinated mice and non-vaccinated mice fed the control diet, suggesting that the weaker impact of T regulatory cells contributes to the augmentation of antitumor immunity by $\mathrm{CD}^{+} \mathrm{T}$ cells in vaccinated mice fed with NYT. Overall, these results indicate that NYT synergistically enhances the effects of the prophylactic tumor vaccine mediated by $\mathrm{CD}^{+} \mathrm{T}$ cells and that this Japanese Kampo medicine has potential as a useful adjuvant agent for cancer immunotherapy.
\end{abstract}

Correspondence to: Dr Shun Takaku, Department of Microbiology and Immunology, Nippon Medical School, 1-1-5 Sendagi, Bunkyo-ku, Tokyo 113-8602, Japan

E-mail: salsa@nms.ac.jp

Key words: ninjin'yoeito, Japanese Kampo medicine, adjuvant, tumor vaccine, prophylactic, cancer immunotherapy

\section{Introduction}

Recent advances in cancer immunotherapy have provided novel treatments for numerous different types of malignancy (1-4). Immune checkpoint therapies, such as antibodies against cytotoxic-T-lymphocyte-associated protein 4 (CTLA-4) and programmed cell death protein 1 (PD-1) that target regulatory pathways in $\mathrm{T}$ cells to enhance antitumor immune responses, have recently generated significant clinical advances in the treatment of cancer $(5,6)$. However, by excessively increasing immune system function, immune checkpoint blockade may lead to inflammatory side effects termed immune-related adverse events (irAEs) (7). Therefore, the safety of cancer immunotherapy requires further improvements.

Although herbal medicines have been widely used in Japan and elsewhere in Asia to treat various types of diseases based on their effects and safety for $\sim 2,000$ years, their mechanisms of action remain unclear. In Japan, patients with cancer are often treated with Japanese traditional herbal medicine (Kampo) in order to palliate a number of symptoms, including fatigue, malaise, anorexia, night sweats, cold limbs and anemia, which may be induced following current standard cancer treatments including surgery, chemotherapy and radiation therapy (8-10). Kampo products generally do not exert direct anticancer effects. However, juzentaihoto (JTT; Shi-Quan-Da-Bu-Tang in Chinese), which comprises ten herbs (Table I), has been reported to prevent the occurrence of cancer, such as estradiol-17 $\beta$-induced endometrial carcinogenesis (11) and melanocytic tumors that spontaneously develop in RET proto-oncogene-transgenic mice (12). In addition, it has been demonstrated to suppress the liver metastasis of CT26-L5 colon carcinoma (13) and lung metastasis of B16 melanoma $(14,15)$ in murine models. Furthermore, JTT was revealed to extend the length of intrahepatic recurrence in patients with hepatocellular carcinoma (16). These results suggest the augmentation of antitumor effects through the use of JTT.

Ninjin'yoeito (NYT; Ren-Shen-Yang-Rong-Tang in Chinese) is another Japanese Kampo medicine that is frequently administered to patients with cancer (17). It comprises twelve herbs (Table I) and is used to treat various symptoms, such as fatigue, anorexia, night sweats, cough, malaise, insomnia, dry skin and dry mouth $(18,19)$. NYT was previously reported to antagonize various forms of anemia, including iron-deficiency (20), aplastic (21), refractory (22) and ribavirin-induced 
anemia in the treatment of chronic hepatitis C (23). However, distinct from JTT, only one study appears to have investigated the ability of NYT to suppress the lung metastasis of CT26-L5 colon carcinoma in mice (24), even though eight herbs are common to NYT and JTT (Table I).

Several previous studies reported that certain Japanese Kampo medicines, including JTT, augment the effects of vaccines (25-30). JTT was demonstrated to synergistically enhance mucosal type 1 immune responses to human papillomavirus in mice orally immunized with a Lactobacillus-based therapeutic human papillomavirus vaccine (29). In humans, the oral administration of JTT increased and prolonged antibody production against A/Victoria/210/2009, particularly following an influenza vaccination (30). Furthermore, JTT was recently demonstrated to exert immune adjuvant effects on tumor vaccine therapy in a subcutaneous (s.c.) OVA-expressing EG7 murine tumor model (31). Therefore, it is postulated that NYT synergistically enhances the effects of antitumor vaccines even though NYT alone does not directly exert any antitumor effects.

In the present study, the antitumor effects of NYT with or without an irradiated tumor cell vaccine were investigated in a prophylactic setting against s.c.-injected CT26 syngeneic colon carcinoma in BALB/c mice to evaluate the potential of NYT as a useful adjuvant for cancer immunotherapy.

\section{Materials and methods}

Mice. Female, inbred BALB/c mice $>6$ weeks old and weighing 16-21 g were purchased from Charles River Japan (Tokyo, Japan). Each experiment comprised 4-8 mice/group. The mice were housed in groups of five in filter cages, and maintained in a temperature-controlled and pathogen-free animal facility with a $14 \mathrm{~h}$ light/10 h dark cycle, with ad libitum access to water and the described diets. All experiments were approved by the Animal Care and Use Committee at Nippon Medical School (Tokyo, Japan).

Diets containing Japanese Kampo medicines. NYT and JTT, comprising twelve and ten herbs, respectively (Table I), were provided as preservative-free pure powders by Tsumura \& Co. (Tokyo, Japan). MF diet (Oriental Yeast Co., Ltd., Tokyo, Japan) was used as a control. Mice were maintained on a control diet, a control diet mixed with NYT (1.0\%; designated NYT diet), or a control diet mixed with JTT (1.0\%; designated JTT diet).

Tumor cell lines. The N-nitro-N-methylurethane-induced $\mathrm{BALB} / \mathrm{c}$ murine colon carcinoma CT26 cell line was purchased from the American Type Culture Collection (ATCC; Manassas, VA, USA) and maintained in RPMI-1640 complete medium (Thermo Fisher Scientific, Inc., Waltham, MA, USA) supplemented with $10 \%$ fetal calf serum (Sigma-Aldrich; Merck KGaA, Darmstadt, Germany), penicillin/streptomycin, L-glutamine, sodium pyruvate, non-essential amino acids, and 2-mercaptoethanol $\left(5 \times 10^{-5} \mathrm{M}\right)$.

Vaccination and tumor inoculation. Irradiated (20,000 rad) CT26 cells $\left(1 \times 10^{5}\right)$ were s.c. injected into various groups of mice. Vaccinated or unvaccinated mice were fed the control,
NYT, or JTT diet from the time of vaccination until four weeks following the inoculation with live tumor cells. Three weeks following the vaccination, $1 \times 10^{6}$ live CT26 cells were s.c. injected into these mice. In certain experiments, vaccinated mice fed the control or NYT diet were intraperitoneally injected with $0.5 \mathrm{mg}$ of the anti-cluster of differentiation (CD) 8 monoclonal antibody (clone. 2.43 , cat. no. TIB-210; ATCC) purified from pooled murine ascites using HiTrap Protein $\mathrm{G}$ HP Columns (GE Healthcare Life Sciences, Chalfont, UK) 1 and 2 days prior to, and 4, 7 and 10 days following the inoculation with live tumor cells.

Flow cytometric analysis with intracellular forkhead box $P 3$ (Foxp3) staining. Regarding the detection of $\mathrm{CD}^{+}, \mathrm{CD}_{25} 5^{+}$and Foxp $3^{+} \mathrm{T}$ cells, spleen cells and tumor-draining lymphocytes were obtained from non-vaccinated mice on the control diet, vaccinated mice on the control diet, and vaccinated mice on the NYT diet on day 28 after the CT26 inoculation. Isolated cells were incubated with $0.5 \mu \mathrm{g}$ of anti-CD16/CD32 (clone, 2.4G2; cat. no. 14-0161; eBioscience, Inc., San Diego, CA, USA) at $4^{\circ} \mathrm{C}$ for $15 \mathrm{~min}$ to reduce nonspecific antibody binding to $\mathrm{Fc}$ receptors. Then, cells were harvested and incubated at $4^{\circ} \mathrm{C}$ for $30 \mathrm{~min}$ with $0.1 \mu \mathrm{g}$ of APC/Cy7-conjugated anti-CD3 (clone, 145-2C11; cat. no. 100329), $0.25 \mu \mathrm{g}$ of FITC-conjugated anti-CD4 (clone, RM4-5; cat. no. 100510), and $0.1 \mu \mathrm{g}$ of APC-conjugated anti-CD25 (clone, PC-61; cat. no. 102011; all BioLegend, Inc., San Diego, CA, USA) for surface staining.

The cells were washed once with $2 \mathrm{ml}$ of cold Dulbecco's phosphate buffered saline (Thermo Fisher Scientific, Inc.) and then fixed with $1 \mathrm{ml}$ of fixation/permeabilization solution (eBioscience) at $4^{\circ} \mathrm{C}$ for $30 \mathrm{~min}$ to allow intracellular staining. Fixed cells were washed twice with $2 \mathrm{ml}$ of $1 \mathrm{X}$ permeabilization buffer (eBioscience) and then stained with $0.4 \mu \mathrm{g}$ of PE-conjugated anti-Foxp3 (clone, FJK-16s; cat. no. 12-5773-80; eBioscience) at $4^{\circ} \mathrm{C}$ for $30 \mathrm{~min}$. After washing twice with $2 \mathrm{ml}$ of $1 \mathrm{X}$ permeabilization buffer, the fluorescence intensity of the stained cells was measured using FACSCanto (BD Biosciences) and the data were analyzed using FlowJo software (version 9.3.1; Tree Star, Inc., Ashland, OR, USA).

Statistical analysis. Data were analyzed using the log-rank test, non-parametric Mann-Whitney U test, or Kruskal-Wallis test with Dunn's multiple comparison post hoc test, using GraphPad Prism 6 software (version 6.0d; GraphPad Software, La Jolla, CA, USA). $\mathrm{P}<0.05$ was considered to indicate a statistically significant difference.

\section{Results}

NYT synergistically enhances whole tumor cell vaccine effects in vivo. In order to clarify whether tumor growth was directly suppressed by NYT in vivo, BALB/c mice were fed the control or NYT diet for three weeks and then inoculated with $1 \times 10^{6}$ live CT26 cells. The mice continued to be fed the same diets until four weeks following the tumor cell inoculation. As illustrated in Fig. 1A, NYT did not affect tumor growth, indicating that NYT alone does not exert any antitumor effects in vivo.

In order to test the hypothesis that NYT acts synergistically with an anticancer vaccine, its antitumor effects were 
Table I. Composition of NYT and JTT.

\begin{tabular}{|c|c|c|c|}
\hline \multirow[b]{2}{*}{ Crude drug } & \multirow[b]{2}{*}{ Botanical origin } & \multicolumn{2}{|c|}{ Ratio, g } \\
\hline & & NYT & JTT \\
\hline Angelicae radix & Root of Angelica acutiloba Kitagawa & 4.0 & 3.0 \\
\hline Poria & Fungus of Poria cocos Wolf & 4.0 & 3.0 \\
\hline Rehmanniae radix & Root of Rehmannia glutinosa Libosch var. purpurea Makino & 4.0 & 3.0 \\
\hline Atractylodis rhizoma & Root of Atractylodes japonica Koidzumi & 4.0 & - \\
\hline Ginseng radix & Root of Panax ginseng C.A. Meyer & 3.0 & 3.0 \\
\hline Cinnamomi cortex & Bark of Cinnamomum cassia Blume & 2.5 & 3.0 \\
\hline Aurantii nobilis pericarpium & Peel of Citrus unshiu Markovich & 2.0 & - \\
\hline Polygalae radix & Root of Polygala tenuifolia Willdenow & 2.0 & - \\
\hline Paeoniae radix & Root of Paeonia lactiflora Pallas & 2.0 & 3.0 \\
\hline Astragali radix & Root of Astragalus membranaceus Bunge & 1.5 & 3.0 \\
\hline Glycyrrhizae radix & Root of Glycyrrhiza uralensis Fisher and DC & 1.0 & 1.5 \\
\hline Schisandrae fructus & Fruit of Schisandra chinensis Baill & 1.0 & - \\
\hline Cnidii rhizoma & Rhizome of Cnidium officinale Makino & - & 3.0 \\
\hline Atractylodis lanceae rhizoma & Rhizome of Atractylodes lancea DC & - & 3.0 \\
\hline
\end{tabular}

NYT, ninjin'yoeito; JTT, juzentaihoto.

evaluated when delivered with a whole-cell vaccine in a prophylactic setting against s.c.-injected CT26 tumors in syngeneic BALB/c mice. The mice were immunized with $1 \times 10^{5}$ irradiated CT26 tumor cells. Certain mice were fed the control or NYT diet for seven weeks from the time of vaccination. Three weeks following vaccination, the mice were challenged with $1 \times 10^{6}$ live CT26 tumor cells. Consistent with our previous findings (32), a longer delay in tumor growth was observed in whole tumor-cell vaccinated mice fed the control diet compared with in non-vaccinated mice fed the control diet (Fig. 1B and C). However, vaccinated mice fed the NYT diet demonstrated significantly more improved protection from tumor growth compared with those fed the control diet $(\mathrm{P}<0.03$; Fig. 1B). Furthermore, the survival rate of vaccinated mice fed the NYT diet was significantly increased compared with that of vaccinated mice fed the control diet $(41.2$ vs. $20.6 \%$; $\mathrm{P}<0.02$; Fig. $1 \mathrm{C}$ ). These results indicate that NYT synergistically enhances antitumor immunity in conjunction with a prophylactic whole-cell vaccine.

Enhancement in tumor vaccine effects through NYT requires $C D 8^{+} T$ cells. In order to elucidate whether $\mathrm{CD}^{+} \mathrm{T}$ cells mediate the enhanced antitumor response observed in vaccinated mice fed the NYT diet, $\mathrm{CD}^{+}$cells were depleted in vivo. As illustrated in Fig. 2, the anti-CD8 treatment in vivo abrogated antitumor effects in vaccinated mice fed NYT. These results indicate that the protection induced by the whole-cell vaccine and NYT is dependent on CD8, as is that induced by the vaccine alone.

Reductions in $\mathrm{CD}^{+}, \mathrm{CD} 25^{+}$and $\mathrm{Foxp}^{+} \mathrm{T}$ regulatory cell numbers in vaccinated mice fed the NYT diet are more apparent than those in vaccinated mice fed the control diet. Since it was previously demonstrated that the depletion of
$\mathrm{CD}^{+}$and $\mathrm{CD}^{2} 5^{+} \mathrm{T}$ regulatory cells enhanced antitumor immunity in the s.c. CT26 tumor model (32), as described by Golgher et al (33), the number of these T regulatory cells in tumor-draining lymph nodes and spleens from different groups of mice were measured on day 28 following the tumor challenge (Fig. 3A and B). In tumor-draining lymph nodes, a significant increase was observed in the $\mathrm{CD} 4^{+}, \mathrm{CD} 25^{+}$and Foxp $3^{+}$T-cell population in non-vaccinated mice fed the control diet. However, the number of these $\mathrm{T}$ regulatory cells was significantly decreased in vaccinated mice, regardless of NYT, compared with in non-vaccinated mice fed the control diet. However, reductions in these cells were more apparent between vaccinated mice fed the NYT diet and non-vaccinated mice fed the control diet than between vaccinated mice and non-vaccinated mice fed the control diet $(\mathrm{P}<0.02$ control vs. vaccine plus control diet group; $\mathrm{P}<0.0002$ control vs. vaccine plus NYT diet group; Fig. 3A). Furthermore, no significant differences were noted in the proportion of $\mathrm{CD} 4^{+}, \mathrm{CD} 25^{+}$and Foxp $3^{+} \mathrm{T}$ regulatory cells in the spleen between vaccinated mice fed the control diet and vaccinated mice fed the NYT diet; however, the number of these cells was significantly decreased in vaccinated mice fed the NYT diet, but not the control diet compared with in non-vaccinated mice fed the control diet $(\mathrm{P}=0.4252$ control vs. vaccine plus control diet group; $\mathrm{P}=0.0095$ control vs. vaccine plus NYT diet group; Fig. 3B). These results indicate that anticancer immunity induced by $\mathrm{CD} 8^{+} \mathrm{T}$ cells may be augmented in vaccinated mice treated with NYT, possibly due to the weaker impact of $\mathrm{T}$ regulatory cells.

JTT does not enhance tumor vaccine efficacy. A recent study reported that JTT exerted immune adjuvant effects for tumor vaccines in another murine tumor model (31). Since eight herbs are common to NYT and JTT (Table I), whether JTT 

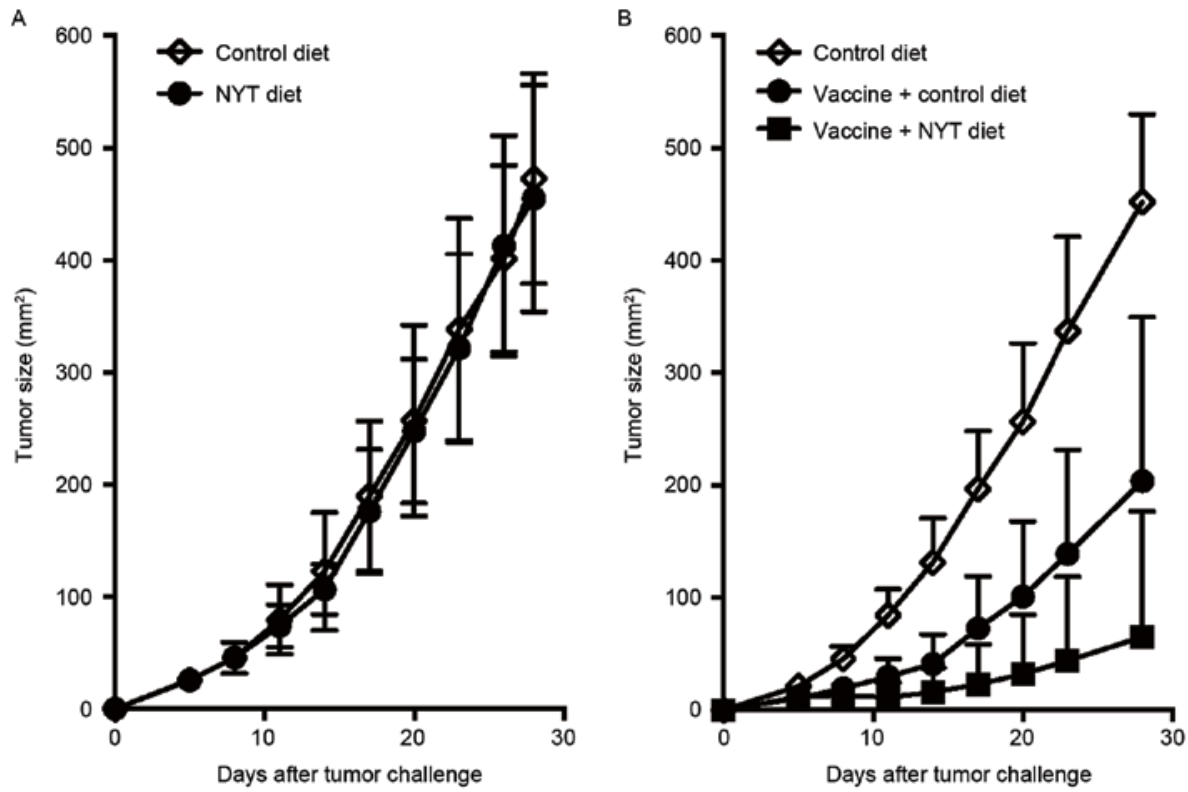

C

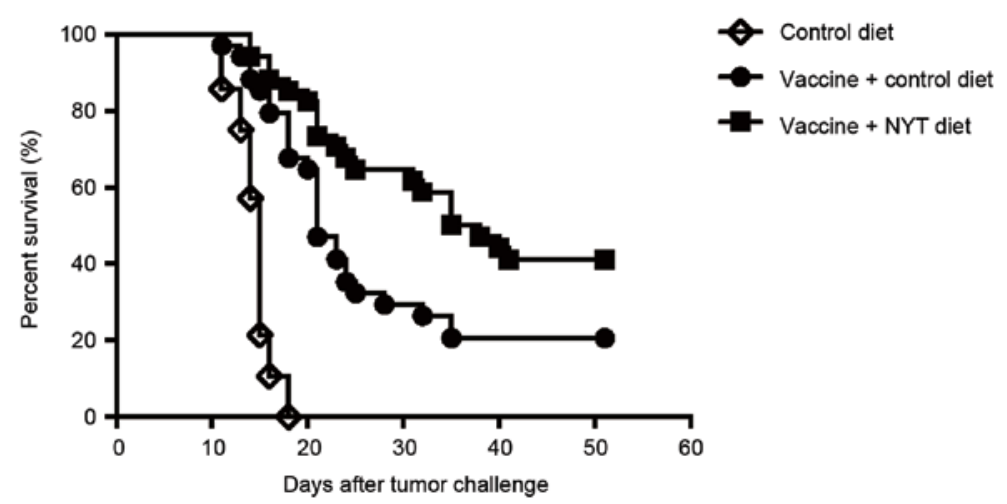

Figure 1. NYT synergistically enhances whole-cell vaccine effects in vivo. (A) Female BALB/c mice were fed the control diet or NYT diet for three weeks and then challenged s.c. with $1 \times 10^{6}$ live syngeneic CT26 cells. These mice continued to be fed the same diets until four weeks following the tumor cell inoculation. Tumors were measured twice weekly using a caliper gauge and their sizes were determined as tumor length $(\mathrm{mm}) \mathrm{x}$ width $(\mathrm{mm})$. Each experiment comprised 4-6 mice/group. These experiments were repeated $\geq 2$ times with similar results. Data are presented as the mean \pm SD. (B) Irradiated (20,000 rad) CT26 cells $\left(1 \times 10^{5}\right)$ were injected s.c. into BALB/c mice. Vaccinated mice were fed the control or NYT diet from the time of the vaccination until four weeks following the tumor cell inoculation. Three weeks following the vaccination, $1 \times 10^{6}$ live CT26 cells were s.c. injected into these mice and tumors were then measured twice weekly. Each experiment included 6-8 mice/group. Results were pooled from two independent experiments. Data are presented as the mean + SD. P $<0.0001$, Mann-Whitney U test between the control diet group and vaccine + control diet group on day 28 following the CT26 inoculation; $\mathrm{P}<0.03$, Mann-Whitney U test between the vaccine + control diet group and vaccine + NYT diet group on day 28 following the tumor cell inoculation. (C) Overall survival rate was compared using the log-rank test. Results were pooled from six independent experiments. A total of 14/34 mice (41.2\%) from the vaccine + NYT diet group remained tumor-free for $\geq 51$ days following the tumor cell inoculation, whereas only $7 / 34$ mice $(20.6 \%)$ in the vaccine + control diet group remained tumor-free in the same time period $(\mathrm{P}<0.02)$. NYT, ninjin'yoeito; s.c., subcutaneously; SD, standard deviation.

also enhanced tumor vaccine efficacy as well as NYT was investigated. The direct antitumor effects exerted by JTT were examined in vivo by taking JTT diet for 3 weeks prior to, and for 4 weeks after the inoculation with $1 \times 10^{6}$ live CT26. As illustrated in Fig. 4A, orally administered JTT without the vaccination did not have any antitumor effects in vivo. It was then examined whether JTT acts synergistically with an anticancer vaccine in a similar manner as that described in Fig. 1B. Although a significantly longer delay was observed in tumor growth in whole tumor-cell vaccinated mice regardless of JTT than in non-vaccinated mice, no significant difference was noted in tumor sizes between the vaccine plus control and vaccine plus JTT diet group (Fig. 4B). These results indicate that unlike NYT, JTT did not function as an adjuvant for the tumor vaccine in the s.c. CT26 tumor model, even though most ingredients are common.

\section{Discussion}

The present study investigated the antitumor effects of NYT combined with or without a whole-cell vaccine in model mice s.c. injected with CT26 tumor cells. NYT alone did not exert any antitumor effects against CT26, whereas a whole-cell vaccine alone significantly delayed tumor growth, as previously described (32). However, the inhibition of tumor growth was significantly greater in vaccinated mice fed the NYT diet 

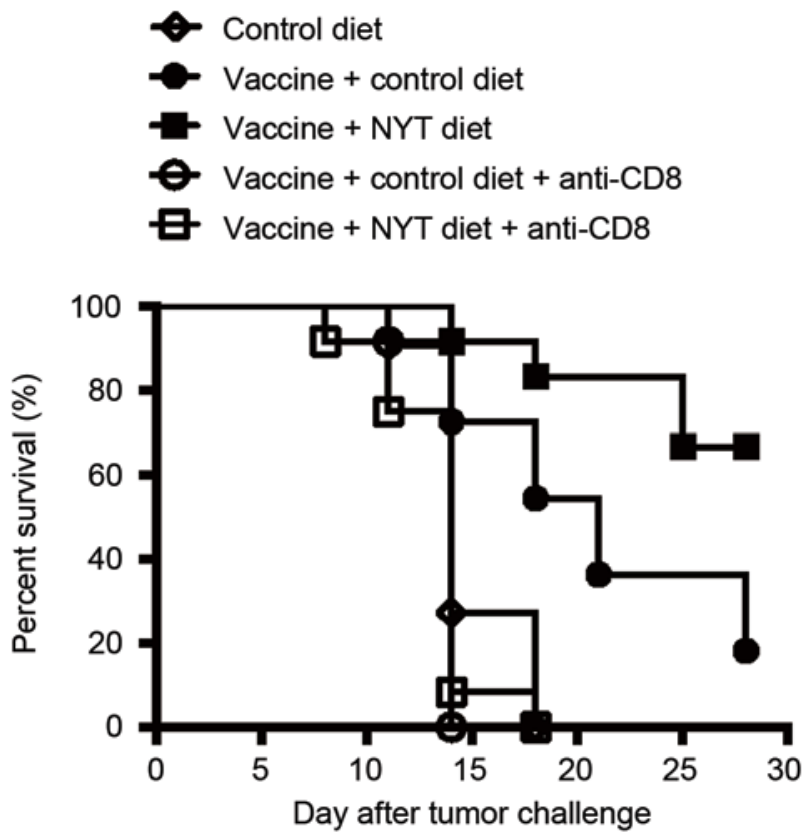

Figure 2. Enhanced antitumor immunity induced by NYT combined with the vaccine is mediated by CD8 ${ }^{+} \mathrm{T}$ cells. Irradiated $(20,000 \mathrm{rad}) \mathrm{CT} 26$ cells $\left(1 \times 10^{5}\right)$ were s.c. injected into BALB/c mice. Vaccinated mice were fed the control or NYT diet from the vaccination until four weeks following the CT26 inoculation. Three weeks following the vaccination, $1 \times 10^{6}$ live CT26 cells were s.c. injected into these mice. Then, 1 and 2 days prior to and 4, 7 , and 10 days following the tumor cell inoculation, certain vaccinated mice were intraperitoneally administered with $0.5 \mathrm{mg}$ of the anti-CD8 monoclonal antibody. Tumors were measured twice weekly. Each experiment included 5-6 mice/group. Results were pooled from two independent experiments $\mathrm{P}<0.0005$, log-rank test between the vaccine + control diet group and vaccine + control diet + anti-CD8 group; $\mathrm{P}<0.0001, \log$-rank test between the vaccine + NYT diet group and vaccine + NYT diet + anti-CD8 group. NYT, ninjin'yoeito; $\mathrm{CD}$, cluster of differentiation; s.c., subcutaneously.

compared with in those fed the control diet. These results suggest that NYT synergistically enhances the effects of the whole-cell vaccine. Since the depletion of $\mathrm{CD}^{+} \mathrm{T}$ cells in vivo resulted in a complete loss of protection from tumors in vaccinated mice treated with NYT, it is suggested that $\mathrm{CD}^{+} \mathrm{T}$ cells mediate the protection.

A previous study reported that regulatory $\mathrm{T}$ cells suppress tumor-specific $\mathrm{CD}^{+}$cytotoxic T-cell cytotoxicity through transforming growth factor (TGF)- $\beta$ signals in vivo, leading to the progression of tumor growth (34). Thus, the number of $\mathrm{CD} 4^{+}, \mathrm{CD} 25^{+}$and $\mathrm{Foxp}^{+} \mathrm{T}$ regulatory cells in various groups of mice was assessed on day 28 following the tumor challenge. No significant difference was observed in the number of these $\mathrm{T}$ regulatory cells between the vaccine plus control diet group and vaccine plus NYT diet group. However, reductions in these cell numbers were more apparent between the vaccine plus NYT diet group and control diet group than between the vaccine plus control diet group and control diet group in tumor-draining lymph nodes $(\mathrm{P}<0.02$, control vs. vaccine plus control diet group; $\mathrm{P}<0.0002$, control vs. vaccine plus NYT diet group), in addition to in spleens ( $\mathrm{P}=0.4252$, control vs. vaccine plus control diet group; $\mathrm{P}=0.0095$, control vs. vaccine plus NYT diet group). Since our previous study revealed that the depletion of $\mathrm{CD} 4{ }^{+} \mathrm{CD} 25^{+} \mathrm{T}$ regulatory cells led to almost complete rejection in s.c. CT26 tumor models (32), as described in other studies $(33,35)$, reductions in $\mathrm{T}$ regulatory cell numbers in vaccinated mice fed NYT may contribute to the enhancements observed in the antitumor effects mediated by $\mathrm{CD}^{+} \mathrm{T}$ cells. Thus, this may lead to additional improved protection from tumor development.

Since eight herbs are common to NYT and JTT, and Takeno et al (31) recently demonstrated the immune adjuvant effects of JTT on tumor vaccine therapy in another murine tumor model, whether JTT also enhances antitumor vaccine effects was investigated in a s.c. CT26 tumor model in the present study. However, JTT did not exhibit any direct antitumor effects and failed to enhance tumor vaccine efficacy. The possibility that immune adjuvant activity in NYT may depend on a difference in the ratio of these eight common ingredients cannot be excluded. Furthermore, these results indicate that the following four herbs: Polygalae radix, Schisandrae fructus, Atractylodis rhizoma and Aurantii nobilis pericarpium, included in NYT, but not in JTT serve an essential role in exerting these immune adjuvant effects. In accordance with this hypothesis, onjisaponin isolated from Polygalae radix has been reported to provide safe and potent adjuvants for the intranasal inoculation of influenza HA, and DPT vaccines by inducing antigen-specific IgA antibodies in nasal washes (36). Furthermore, the topical application of gomisin A, a dibenzocyclooctadiene lignan isolated from Schisandara fructus, inhibited tumor promotion by 12 -O-tetradecanoylphorbol-13-acetate in two-stage carcinogenesis of mouse skin cells (37). Therefore, further studies are required in order to elucidate whether onjisaponin and gomisin A perform essential roles in the enhanced anticancer vaccine effects observed in the murine tumor model used in the current study.

NYT has also been reported to inhibit the development of proteinuria and prolong the survival of MRL/lpr mice that develop a systemic lupus erythematosus-like autoimmune disease including autoimmune nephritis, possibly through the suppression of active TGF- $\beta$ (38). Since our previous study revealed that inhibiting TGF- $\beta$ using an anti-TGF- $\beta$ antibody synergistically enhanced the effects of a tumor-cell vaccine mediated by $\mathrm{CD}^{+} \mathrm{T}$ cells $(32,39)$, NYT may similarly suppress the active form of TGF- $\beta$ in vaccinated mice, leading to more improved protection from tumor growth. These issues warrant further investigation.

Immune checkpoint inhibitors, such as the anti-CTLA-4 antibody and anti-PD-1 antibody have recently led to significant clinical advances in cancer immunotherapy $(5,6)$. However, safety concerns have been expressed as CTLA-4 and PD-1 are included in co-inhibitory molecules and a disruption in the functioning of these immune checkpoint molecules may cause imbalances in immunological tolerance that result in an unchecked immune response, ultimately leading to irAEs $(7,40)$. Furthermore, these agents are usually expensive $(41,42)$. However, traditional herbal medicines, including Kampo medicine, have been selected from numerous primitive phytomedicines and developed based on their effects, and safety over a period of 2,000 years in East Asia, including Japan (43). Therefore, these medicines are considered to be essentially free of deleterious side effects; however, reliable statistical evidence from large-scale controlled studies to support this assertion is limited or absent. In accordance with these notions, no adverse effects were identified in the 


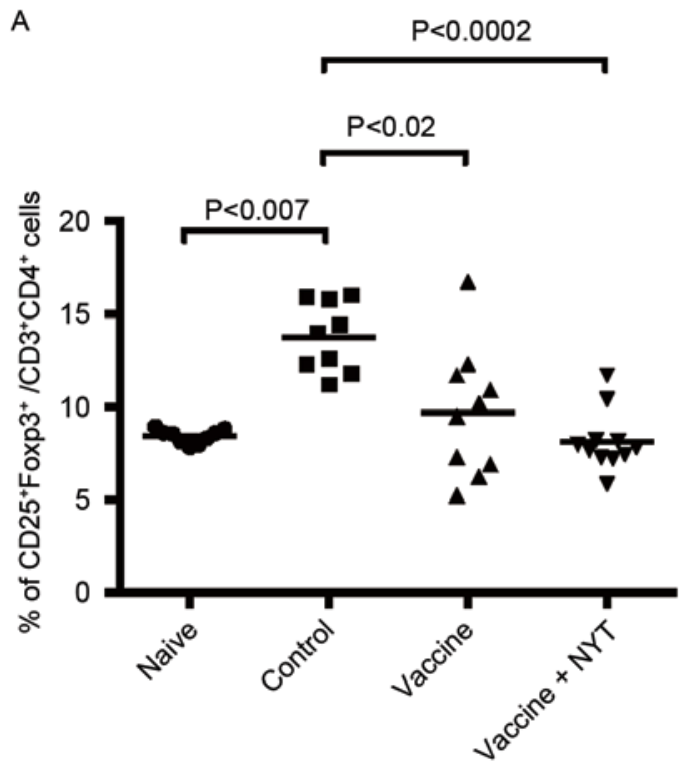

Groups of mice
B
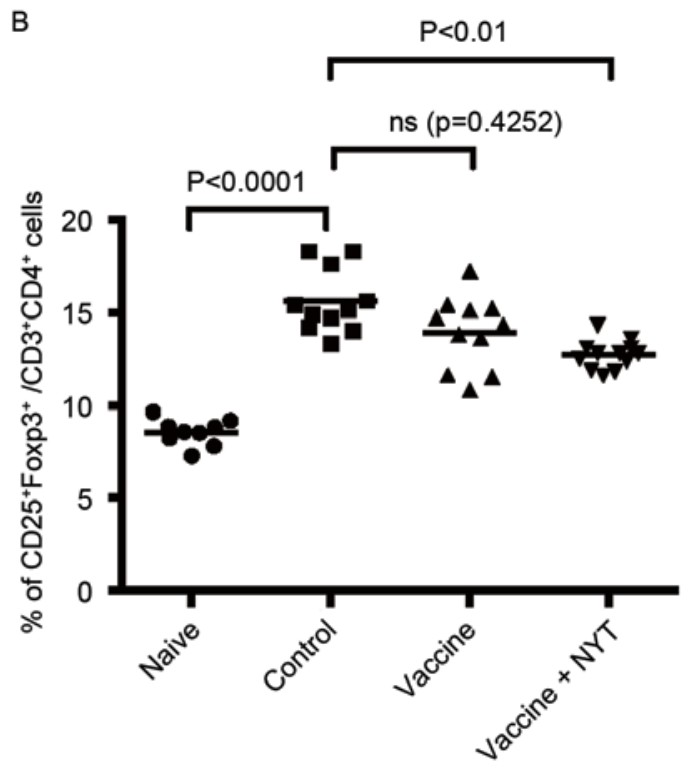

Groups of mice

Figure 3. Reductions in $\mathrm{CD}^{+}, \mathrm{CD} 25^{+}$and $\mathrm{Foxp}_{3}{ }^{+} \mathrm{T}$ regulatory cell numbers in the vaccine with $\mathrm{NYT}$ diet group are more apparent compared with those in the vaccine with control diet group. Irradiated $(20,000 \mathrm{rad})$ CT26 cells $\left(1 \times 10^{5}\right)$ were s.c. injected into BALB/c mice. Vaccinated mice were fed the control diet or NYT diet from the vaccination until four weeks following the tumor challenge. Three weeks following the vaccination, $1 \times 10^{6}$ live CT26 cells were s.c. injected into these mice. Twenty-eight days following the tumor challenge, (A) tumor-draining lymph nodes and (B) spleens were recovered and stained with anti-CD3, anti-CD4, anti-CD25 and anti-Foxp3. The proportions of $\mathrm{CD}^{+}, \mathrm{CD}^{+}, \mathrm{CD} 25^{+}$and Foxp3 ${ }^{+}$cells were determined using flow cytometry. Four to six female mice were used for each group. Results were pooled from two independent experiments. Each symbol represents one data point; the bars are the median. All P-values were calculated using the Kruskal-Wallis test with Dunn's multiple comparison test. The naïve group contained mice that received no vaccination or tumor challenge. NYT, ninjin'yoeito; CD, cluster of differentiation; foxp3, forkhead box P3; s.c., subcutaneously.
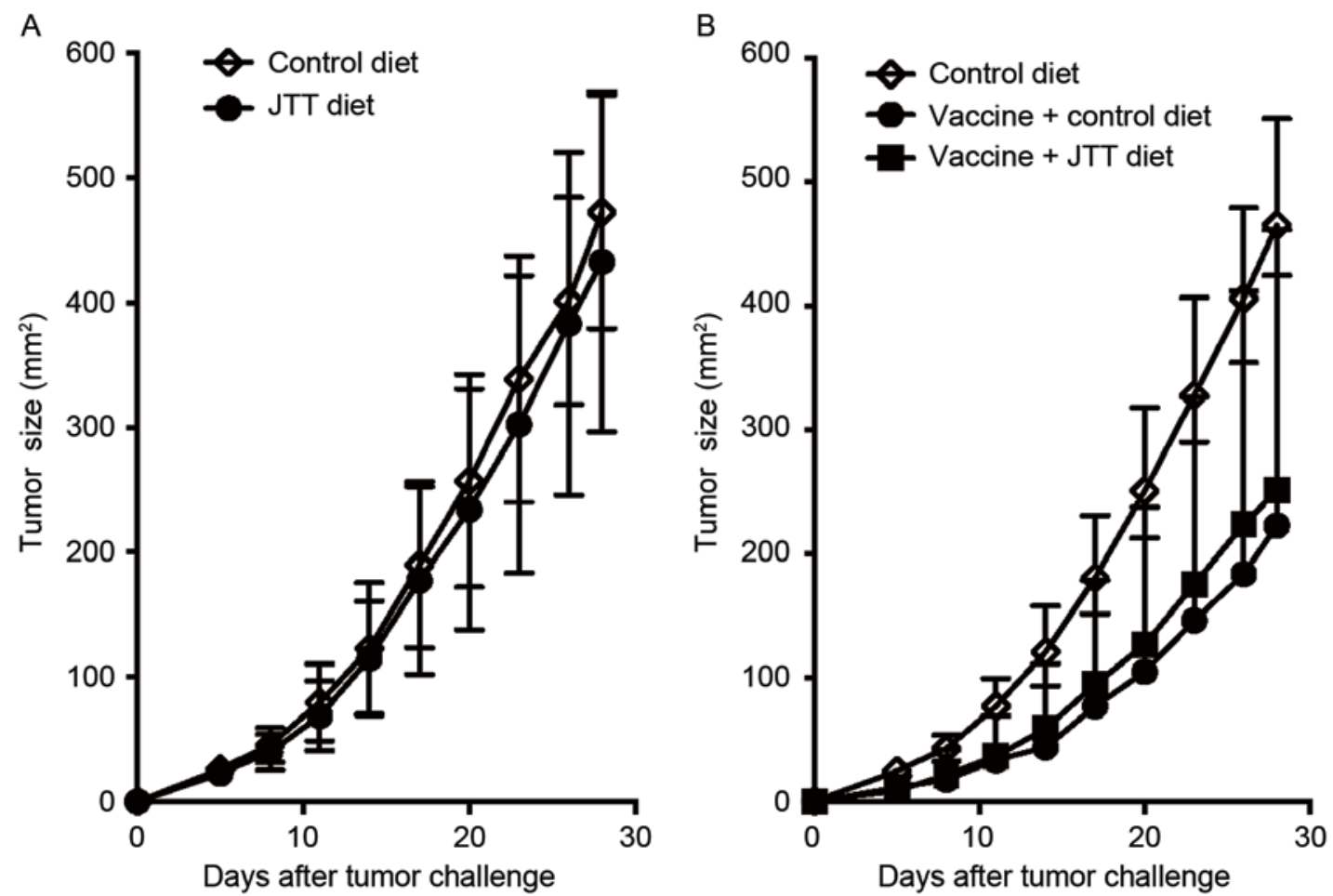

Figure 4. JTT does not enhance tumor vaccine efficacy. Female BALB/c mice were fed the control or JTT diet for three weeks and then challenged s.c. with $1 \times 10^{6}$ live syngeneic CT26 cells. These mice continued to be fed the same diets until four weeks following the tumor challenge. (A) Tumors were measured twice weekly. Each experiment comprised four to six mice per group. These experiments were repeated at least twice with similar results. The results are presented as the mean \pm SD. (B) Irradiated $(20,000 \mathrm{rad})$ CT26 cells $\left(1 \times 10^{5}\right)$ were injected s.c. into BALB/c mice. Vaccinated mice were fed the control diet or JTT diet from the time of the vaccination until four weeks following the tumor challenge. Three weeks following the vaccination, $1 \times 10^{6}$ live CT26 cells were s.c. injected into these mice and tumors were then measured twice weekly. Each experiment included five to six mice per group. Results were pooled from two independent experiments. The results are presented as the mean + SD. $\mathrm{P}<0.01$, Mann-Whitney test between the control diet group and vaccine plus control diet group on day 28 following the CT26 challenge; $\mathrm{P}<0.02$, Mann-Whitney test between the control diet group and vaccine plus JTT diet group on day 28 following the CT26 challenge. s.c., subcutaneously; JTT, juzentaihoto; SD, standard deviation. 
mice fed on the NYT diet in the present study. In addition, various Kampo products have been available under the Japanese National Health Insurance System since 1976 and are cost-effective (43). The daily cost of NYT is $\sim 0.4 \%$ that of immune checkpoint inhibitors (44). Therefore, NYT has potential as a relatively safe and more cost-effective adjuvant agent for enhancing antitumor immunity.

In conclusion, to the best of our knowledge, this is the first study to demonstrate synergistic enhancements in $\mathrm{CD}^{+}$ $\mathrm{T}$ cell-mediated tumor vaccine efficacy by the oral administration of NYT. Although the underlying mechanism for the augmented anticancer vaccine effects induced by NYT have yet to be fully elucidated, the current study, in conjunction with previous findings on JTT presented by Takeno et al (31), provide a novel insight into the use of Japanese Kampo medicines as tumor vaccine adjuvants.

\section{Acknowledgements}

The present study was supported by the Ministry of Education, Culture, Sports, Science, and Technology (MEXT) KAKENHI, Japan (grant no. 24501335; received by S.T.).

\section{References}

1. Kochenderfer JN, Dudley ME, Kassim SH, Somerville RP, Carpenter RO, Stetler-Stevenson M, Yang JC, Phan GQ, Hughes MS, Sherry RM, et al: Chemotherapy-refractory diffuse large B-cell lymphoma and indolent B-cell malignancies can be effectively treated with autologous T cells expressing an anti-CD19 chimeric antigen receptor. J Clin Oncol 33: 540-549, 2015.

2. Robert C, Long GV, Brady B, Dutriaux C, Maio M, Mortier L, Hassel JC, Rutkowski P, McNeil C, Kalinka-Warzocha E, et al: Nivolumab in previously untreated melanoma without BRAF mutation. N Engl J Med 372: 320-330, 2015.

3. Rizvi NA, Mazières J, Planchard D, Stinchcombe TE, Dy GK, Antonia SJ, Horn L, Lena H, Minenza E, Mennecier B, et al Activity and safety of nivolumab, an anti-PD-1 immune checkpoint inhibitor, for patients with advanced, refractory squamous non-small-cell lung cancer (CheckMate 063): A phase 2, single-arm trial. Lancet Oncol 16: 257-265, 2015.

4. Ansell SM, Lesokhin AM, Borrello I, Halwani A, Scott EC, Gutierrez M, Schuster SJ, Millenson MM, Cattry D, Freeman GJ, et al: PD-1 blockade with nivolumab in relapsed or refractory Hodgkin's lymphoma. N Engl J Med 372: 311-319, 2015.

5. Sharma P and Allison JP: The future of immune checkpoint therapy. Science 348: 56-61, 2015.

6. Postow MA, Callahan MK and Wolchok JD: Immune checkpoint blockade in cancer therapy. J Clin Oncol 33: 1974-1982, 2015.

7. Postow MA: Managing immune checkpoint-blocking antibody side effects. Am Soc Clin Oncol Educ Book: 76-83, 2015.

8. Amitani M, Amitani H, Sloan RA, Suzuki H, Sameshima N, Asakawa A, Nerome Y, Owaki T, Inui A and Hoshino E: The translational aspect of complementary and alternative medicine for cancer with particular emphasis on Kampo. Front Pharmacol 6: 150, 2015.

9. Yamakawa J, Motoo Y, Moriya J, Ogawa M, Uenishi H, Akazawa S, Sasagawa T, Nishio M and Kobayashi J: Role of Kampo medicine in integrative cancer therapy. Evid Based Complement Alternat Med 2013: 570848, 2013.

10. Horie Y, Kato K, Kameoka S and Hamano K: Bu ji (hozai) for treatment of postoperative gastric cancer patients. Am J Chin Med 22: 309-319, 1994

11. Niwa K, Hashimoto M, Morishita S, Lian Z, Tagami K, Mori $\mathrm{H}$ and Tamaya T: Preventive effects of Juzen-taiho-to on $\mathrm{N}$-methyl-N-nitrosourea and estradiol-17beta-induced endometrial carcinogenesis in mice. Carcinogenesis 22: 587-591, 2001.

12. Dai Y, Kato M, Takeda K, Kawamoto Y, Akhand AA, Hossain K, Suzuki H and Nakashima I: T-cell-immunity-based inhibitory effects of orally administered herbal medicine juzen-taiho-to on the growth of primarily developed melanocytic tumors in RET-transgenic mice. J Invest Dermatol 117: 694-701, 2001.
13. Ohnishi Y, Fujii H, Hayakawa Y, Sakukawa R, Yamaura T, Sakamoto T, Tsukada K, Fujimaki M, Nunome S, Komatsu Y and Saiki I: Oral administration of a Kampo (Japanese herbal) medicine Juzen-taiho-to inhibits liver metastasis of colon 26-L5 carcinoma cells. Jpn J Cancer Res 89: 206-213, 1998.

14. Onishi Y, Yamaura T, Tauchi K, Sakamoto T, Tsukada K, Nunome S, Komatsu Y and Saiki I: Expression of the anti-metastatic effect induced by Juzen-taiho-to is based on the content of Shimotsu-to constituents. Biol Pharm Bull 21: 761-765, 1998.

15. Matsuda T, Maekawa K, Asano K and Hisamitsu T: Suppressive effect of juzen-taiho-to on lung metastasis of b16 melanoma cells in vivo. Evid Based Complement Alternat Med 2011: 743153 , 2011.

16. Tsuchiya M, Kono H, Matsuda M, Fujii H and Rusyn I: Protective effect of Juzen-taiho-to on hepatocarcinogenesis is mediated through the inhibition of Kupffer cell-induced oxidative stress. Int J Cancer 123: 2503-2511, 2008.

17. Kamei T, Kumano H, Iwata K, Nariai Y and Matsumoto T: The effect of a traditional Chinese prescription for a case of lung carcinoma. J Altern Complement Med 6: 557-559, 2000.

18. Scheid V, Bensky D, Ellis A and Barolet R: Chinese Herbal Medicine Formulas \& Strategies. Eastland Press, Inc., Seattle, WA, pp348-352, 2009.

19. Suzuki T, Yamamoto A, Ohsawa M, Motoo Y, Mizukami H and Makino T: Ninjin'yoeito and ginseng extract prevent oxaliplatin-induced neurodegeneration in PC12 cells. J Nat Med 69: 531-537, 2015.

20. Ando N: Blood making effect of Ninjin-yoei-to (Ren-shenyang-rong-tang) as monotherapy in obesteric and gynecologic patient with anemia. Jpn J Orient Med 50: 461-470, 1999.

21. Miyazaki T, Uchino H, Kimura I, Saito H, Shibata A, Takaku F, Nagai K, Niho Y, Matsuda T, Miura A, et al: Effects of Ren-shen-yang-rong-tang (Japanese name: Ninjin-youei-to), a traditional herbal medicine, on the hematopoiesis in the patients with aplastic anemia. J Clin Ther Med 10: 2591-2603, 1994 (In Japanese).

22. Ohmori S, Usui T, Yasuda N, Ohmori M, Yamagishi M and Okuma M: Improvement of anemia by administration of EK-108 [Chinese herbal medicine, Ren-Shen-Yang-Rong-Tang (Japanese name, Ninjin-Yoei-To)] in a patient of myelodisplastic syndrome. Jpn Pharmacol Ther 20: 361-365, 1992.

23. Motoo Y, Mouri H, Ohtsubo K, Yamaguchi Y, Watanabe H and Sawabu N: Herbal medicine Ninjinyoeito ameliorates ribavirin-induced anemia in chronic hepatitis C: A randomized controlled trial. World J Gastroenterol 11: 4013-4017, 2005.

24. Matsuo M, Tani T and Saiki I: Organ selectivity of Juzen-taiho-to and Ninjin-yoei-to in the expression of anti-metastatic efficacy. J Trad Med 19: 93-97, 2002.

25. Kiyohara H, Nagai T, Munakata K, Nonaka K, Hanawa T, Kim SJ and Yamada H: Stimulating effect of Japanese herbal (kampo) medicine, hochuekkito on upper respiratory mucosal immune system. Evid Based Complement Alternat Med 3: 459-467, 2006.

26. Matsumoto T, Noguchi M, Hayashi O, Makino K and Yamada H: Hochuekkito, a Kampo (traditional Japanese herbal) medicine, enhances mucosal IgA antibody response in mice immunized with antigen-entrapped biodegradable microparticles. Evid Based Complement Alternat Med 7: 69-77, 2010.

27. Underwood JR, Chivers M, Dang TT and Licciardi PV: Stimulation of tetanus toxoid-specific immune responses by a traditional Chinese herbal medicine. Vaccine 27: 6634-6641, 2009.

28. Licciardi PV and Underwood JR: Identification of a novel vaccine adjuvant that stimulates and maintains diphtheria toxoid immunity. Vaccine 28: 3865-3873, 2010.

29. Taguchi A, Kawana K, Yokoyama T, Adachi K, Yamashita A, Tomio K, Kojima S, Oda K, Fujii T and Kozuma S: Adjuvant effect of Japanese herbal medicines on the mucosal type 1 immune responses to human papillomavirus (HPV) E7 in mice immunized orally with Lactobacillus-based therapeutic HPV vaccine in a synergistic manner. Vaccine 30: 5368-5372, 2012.

30. Saiki I, Koizumi K, Goto H, Inujima A, Namiki T, Raimura M, Kogure T, Tatsumi T, Inoue H, Sakai S, et al: The long-term effects of a kampo medicine, juzentaihoto, on maintenance of antibody titer in elderly people after influenza vaccination. Evid Based Complement Alternat Med 2013: 568074, 2013.

31. Takeno N, Inujima A, Shinohara K, Yamada M, Shibahara N, Sakurai H, Saiki I and Koizumi K: Immune adjuvant effect of Juzentaihoto, a Japanese traditional herbal medicine, on tumor vaccine therapy in a mouse model. Int J Oncol 47: 2115-2122, 2015. 
32. Takaku S, Terabe M, Ambrosino E, Peng J, Lonning S, McPherson JM and Berzofsky JA: Blockade of TGF-beta enhances tumor vaccine efficacy mediated by CD8(+) T cells. Int J Cancer 126: 1666-1674, 2010.

33. Golgher D, Jones E, Powrie F, Elliott T and Gallimore A: Depletion of CD25+ regulatory cells uncovers immune responses to shared murine tumor rejection antigens. Eur J Immunol 32: 3267-3275, 2002.

34. Chen ML, Pittet MJ, Gorelik L, Flavell RA, Weissleder R, von Boehmer $\mathrm{H}$ and Khazaie K: Regulatory $\mathrm{T}$ cells suppress tumor-specific CD8 T cell cytotoxicity through TGF-beta signals in vivo. Proc Natl Acad Sci USA 102: 419-424, 2005.

35. Casares N, Arribillaga L, Sarobe P, Dotor J, Lopez-Diaz de Cerio A, Melero I, Prieto J, Borrás-Cuesta F and Lasarte JJ: CD4+/CD25+ regulatory cells inhibit activation of tumor-primed CD4+ T cells with IFN-gamma-dependent antiangiogenic activity, as well as long-lasting tumor immunity elicited by peptide vaccination. J Immunol 171: 5931-5939, 2003

36. Nagai T, Suzuki Y, Kiyohara H, Susa E, Kato T, Nagamine T, Hagiwara Y, Tamura S, Yabe T, Aizawa $\mathrm{C}$ and Yamada $\mathrm{H}$ : Onjisaponins, from the root of Polygala tenuifolia Willdenow, as effective adjuvants for nasal influenza and diphtheriapertussis-tetanus vaccines. Vaccine 19: 4824-4834, 2001.

37. Yasukawa K, Ikeya Y, Mitsuhashi H, Iwasaki M, Aburada M, Nakagawa S, Takeuchi M and Takido M: Gomisin A inhibits tumor promotion by 12-O-tetradecanoylphorbol-13-acetate in two-stage carcinogenesis in mouse skin. Oncology 49: 68-71, 1992.
38. Nakai S, Kawakita T, Himeno $\mathrm{K}$ and Nomoto K: Combined treatments with Ninjin-youei-to (Ren-shen-yang-rong-tang) plus a suboptimal dose of prednisolone on autoimmune nephritis in MRL/lpr mice. Int J Immunopharmacol 20: 275-284, 1998.

39. Terabe M, Ambrosino E, Takaku S, O'Konek JJ, Venzon D, Lonning S, McPherson JM and Berzofsky JA: Synergistic enhancement of CD8+ T cell-mediated tumor vaccine efficacy by an anti-transforming growth factor-beta monoclonal antibody. Clin Cancer Res 15: 6560-6569, 2009.

40. Naidoo J, Page DB, Li BT, Connell LC, Schindler K, Lacouture ME, Postow MA and Wolchok JD: Toxicities of the anti-PD-1 and anti-PD-L1 immune checkpoint antibodies. Ann Oncol 26: 2375-2391, 2015.

41. Medina PJ and Adams VR: PD-1 pathway inhibitors: Immuno-oncology agents for restoring antitumor immune responses. Pharmacotherapy 36: 317-334, 2016.

42. Choudhury N and Nakamura Y: Importance of immunopharmacogenomics in cancer treatment: Patient selection and monitoring for immune checkpoint antibodies. Cancer Sci 107: 107-115, 2016.

43. Saiki I: A Kampo medicine 'Juzen-taiho-to'-prevention of malignant progression and metastasis of tumor cells and the mechanism of action. Biol Pharm Bull 23: 677-688, 2000.

44. Takaku F, Yazaki Y, Kitahara M, Ueno F and Echizen H: Manual of Therapeutic Agents. Igaku-shoin, Inc., Tokyo, Japan, pp2322, 2016. 